報文 3

\title{
Flash Photolysis of Aromatic Thiols in Aqueous Solution
}

\author{
'Takeshi Takakura, Koichi Fujioka, Masaki OKuyama \\ and Hitoshi KAMADA \\ Department of Industrial Chemistry, Faculty of Engineering, \\ The University of Tokyo, Hongo, Bunkyo-ku, Tokyo 113
}

(Received September 16, 1975)

高倉毅, 藤 岡 宏一,
奥山正喜, 鎌田仁

東京大学工学部工業化学科, 東京都文京区本郷（干 113）

\begin{abstract}
The flash photolysis of thiophenol and its derivatives in aqueous solutions produced the transients which had the strong absorptions with maxima at $470 \sim 600 \mathrm{~nm}$. The transients were assigned to the disulfide anion radicals. The decay of the radicals was first-order and the decay constants of some radicals were determined. It was suggested that the transition corresponding to the observed spectra should involve $\pi$-electron of rings.
\end{abstract}

\section{Introduction}

In the preceding paper ${ }^{1)}$, we have studied the flash photolysis of thiophenols in organic solvents. The observed transient spectra which had maxima at $\sim 500 \mathrm{~nm}$ and strong continuous absorption bands below $\sim 400 \mathrm{~nm}$ have been attributed to the thiophenoxy radicals.

On the other hand, Caspari and Granzow ${ }^{2)}$ have observed a transient spectrum with a maximum at $470 \mathrm{~nm}$ by the flash photolysis of thiophenol in aqueous solution, and identified it as arising from the diphenyl disulfide anion radical. They have proposed that the formation of the anion radical from flash photolysis was via the fast reaction of the primary $\mathrm{PhS}$. radical with a $\mathrm{PhS}^{-}$anion.

$$
\begin{aligned}
& \mathrm{PhSH} \rightleftarrows \mathrm{PhS}^{-}+\mathrm{H}^{+} \\
& \mathrm{PhS}^{-} \stackrel{h_{\nu}}{\rightarrow} \mathrm{PhS} \cdot+\mathrm{e}_{\mathrm{aq}} \\
& \mathrm{PhS} \cdot+\mathrm{PhS}^{-} \rightleftarrows \mathrm{PhSSPh}^{-} \rightarrow \text { product }
\end{aligned}
$$

As described in the preceding paper, there are some confusion and question on the assignment of the transient spectra observed from the flash photolysis of thiophenols in organic solvents and in aqueous solution. We therefore conducted the flash photolysis of thiophenol and its derivatives in aqueous solution in order to obtain more detailed information on the transients.

\section{Experimental}

The flash photolysis apparatus used in this work has been described elsewhere ${ }^{1)}$. Experiments were made mostly in alkaline solutions $(\mathrm{pH}=12 \sim 13)$ and partly in acidic solutions. Solutions were prepared just prior to use and degassed by freeze-pump-thaw cycles. A fresh solution was used for each photolytic flash delivered. Materials were of research grade and used as received. 


\section{Results and Discussion}

\subsection{Transient spectra}

Thiols dissociate into a thiyl anion and a proton in aqueous solution, especially in alkaline solution. Figure 1 shows the $\mathrm{pH}$ dependence of the UV spectra of $p$-chlorothiophenol. Undissociated $p$-chlorothiophenol has an absorption maximum at $245 \mathrm{~nm}$, and the absorption of the dissociated anion (maximum at $272 \mathrm{~nm}$ ) increases with increasing the $\mathrm{pH}$ of the solution. Since the $\mathrm{p} K_{\mathrm{a}}$ of $p$-chlorothiophenol is $7.8^{3)}$, it is almost dissociated to the anion at $\mathrm{pH}=11$. In what follows, the transients observed in alkaline solutions will be described.

Figure 2 shows the transient spectra observed from thiophenol and thiocresols. The spectrum from thiophenol (Fig. 2a) is in agreement with the result of Caspari and Granzow ${ }^{2}$. The spectrum has a broad peak at $470 \mathrm{~nm}$ and is obviously different from the spectrum of the thiophenoxy radical which, as shown in the preceding paper, is obtained from the flash

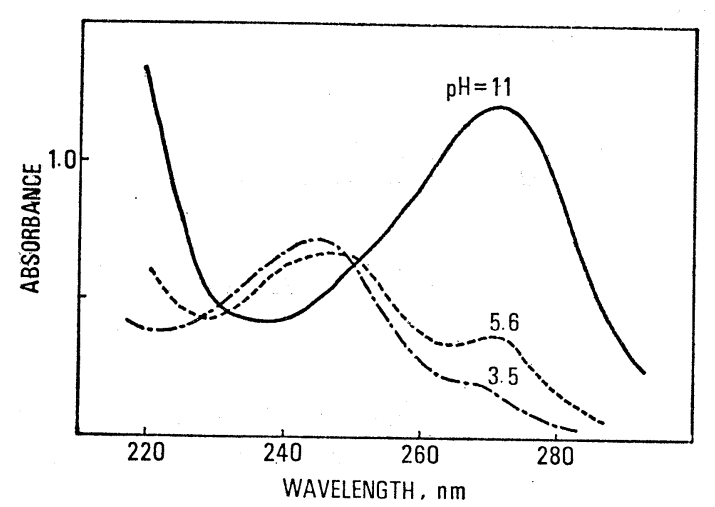

Fig. 1. The pH dependence of tne UV absorption spectra of $p$-chlorothiophenol in aqueous solution.

photolysis of thiophenol in organic solvents. The spectra from thiocresols (Figs. $2 \mathrm{~b} \sim 2 \mathrm{~d}$ ) are similar to the spectrum from thiophenol although the peak positions and intensities are slightly different. Consequently, it is almost certain that all the spectra from thiophenol and thiocresols shown in Fig. 2 are attributed

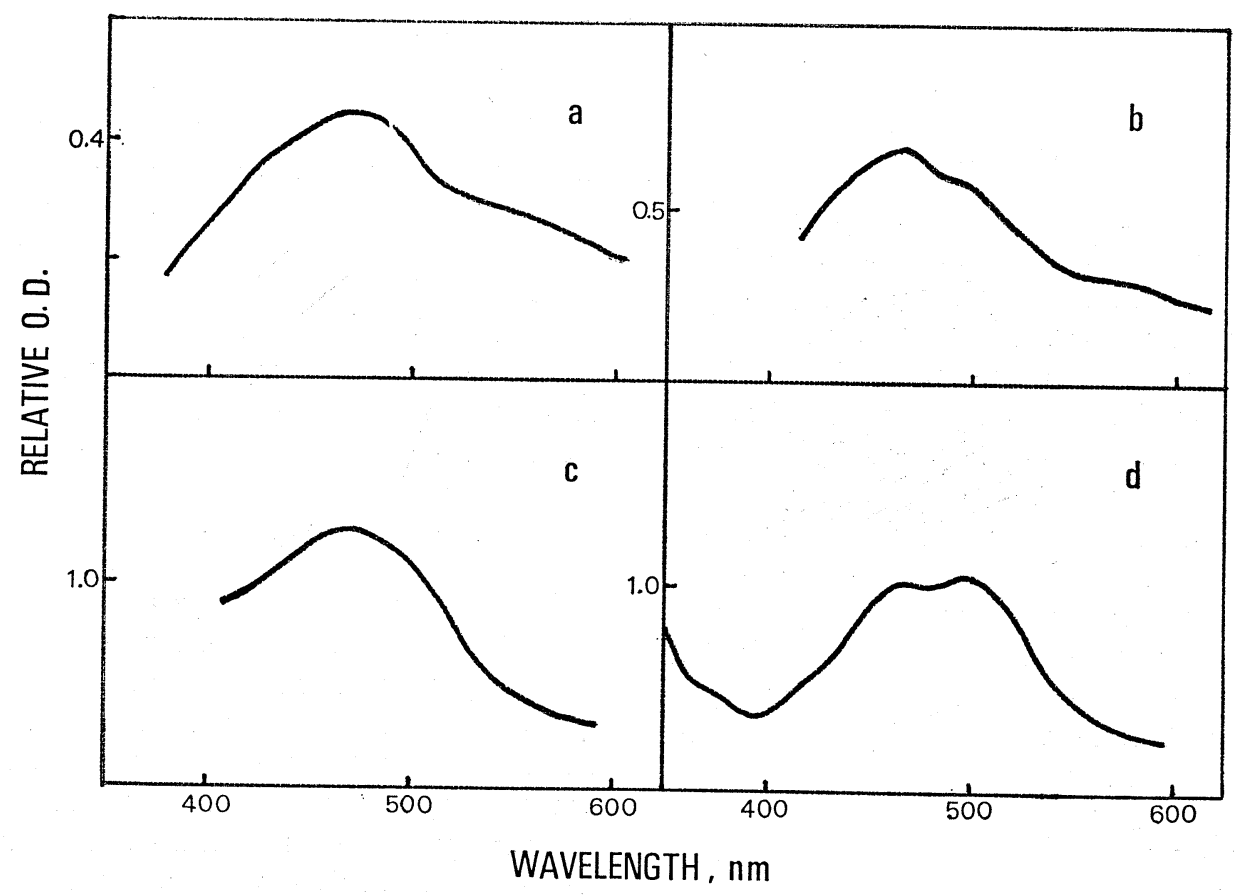

Fig. 2. Transient absorption spectra in alkaline solutions. Observed from; (a) thiophenol, (b) $o$-thiocresol, (c) $m$-thiocresol and (d) $p$-thiocresol. 


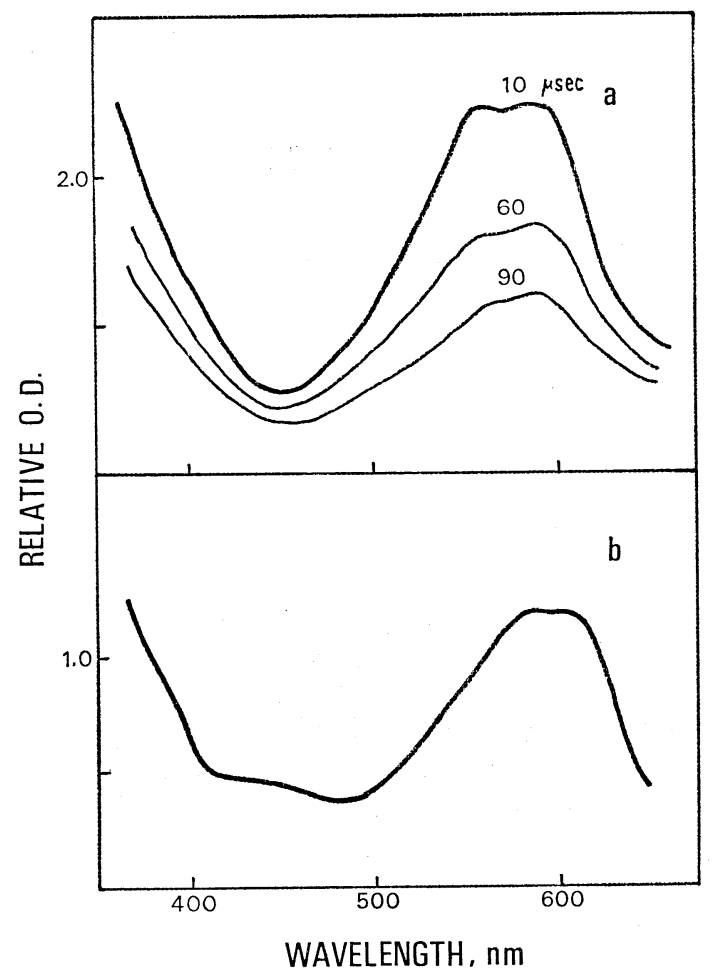

Fig. 3. Transient absorption spectra in alkaline solutions. Observed from; (a) p-chlorothiophenol and (b) p-aminothiophenol.

to the same kind of transients.

On the other hand, the peak positions shift by $c a .100 \mathrm{~nm}$ to longer wavelengths and the intensities increase by about one order of magnitude in the cases of the spectra from $p$-chlorothiophenol and $p$-aminothiophenol (Fig. $3)$, compared with the spectra from thiophenol and thiocresols. As the spectral changes are rather large, it is probable that these spectra are attributed to another kind of transients. However, the decay of the spectrum from $p$ chlorothiophenol is simple, as shown in Fig. $3 a$, and the decay constant of the transient from $p$-chlorothiophenol is nearly equal to that from $p$-thiocresol of Fig. 2 (vide infra). Therefore, we assumed that all of the transients are of the same kind.

Since thiophenols dissociate into an anion and a proton in alkaline solution, it is impossible for them to undergo the radical fission of the $\mathrm{S}-\mathrm{H}$ bond. Accordingly the primary process is supposed to be the formation of the

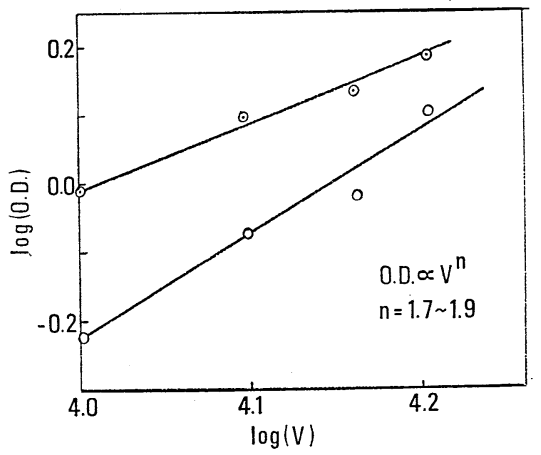

Fig. 4. The dependence of the optical density of the transient absorption spectrum (observed from $p$-chlorothiophenol) on the discharge voltage of the photolytic flash. Observed at $540 \mathrm{~nm}(\odot)$ and $620 \mathrm{~nm}(\bigcirc)$.

thiophenoxy radicals by photodetachment of an electron. Nevertheless, the observed spectra are different from the spectra of the thiophenoxy radicals as stated above. It is therefore most reasonable that the observed transients are disulfide anion radicals formed by reaction (3), as proposed by Caspari and Granzow.

The relationship between the discharge voltage of photolytic flash and the absorption intensity of the transient spectrum from $p$ chlorothiophenol was measured in order to determine whether the photodetachment of an electron (reaction (2)) is one or two photon process (Fig. 4). The intensity was nearly proportional to the second power of the dis-

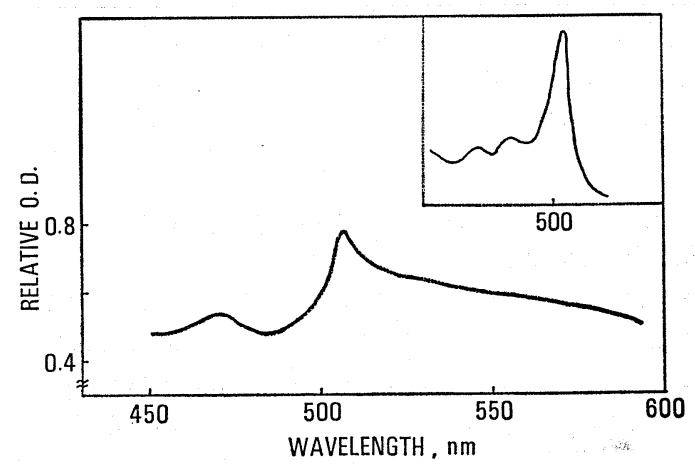

Fig. 5. Transient absorption spectrum observed from $p$-chlorothiophenol in acidic solution. Inset shows the transieut spectrum observed in cyclohexane ( $p$-chlorothiophenoxy radical). 
charge voltage, i.e., the first power of the light intensity, and hence the photodetachment was found to be one photon process.

Figure 5 shows the transient spectrum observed from $p$-chlorothiophenol in acidic solution. This is distinct from the spectrum in alkaline solution, and it is concluded that the transient is $p$-chlorothiophenoxy radical (see inset). The suggestion by Caspari and Granzow $^{2)}$ that the anion radical is produced in neutral or acidic solution through photo. dissociation of thiophenol may be incorrect.

\subsection{Decay kinetics}

The decay of the spectra observed from thiophenol, $p$-thiocresol and $p$-chlorothiophenol was examined as a function of time (delay time of the analysing flash). The intensities of the spectra were followed only for 100 or $150 \mu \mathrm{sec}$ after the photolytic flash. The intensities did not apparently decay at longer delay times because the produced disulfide were insoluble in water. The decay was substantially first-order in all cases. The decay constants, $k$, and life times are shown in Table I. Caspari and Granzow ${ }^{2}$ have reported a value $k=3.8 \times 10^{4}$ at $\mathrm{pH}=10.6$ with respect to thiophenol and our result is slightly smaller than their value. An anion radical may be stabilized by a para substitution as shown in Table I.

Table I. The decay constants of disulfide anion radicals.

\begin{tabular}{l|c|c}
\hline \multicolumn{1}{c|}{ reactant } & $k\left(\mathrm{sec}^{-1}\right)$ & life time $(\mu \mathrm{sec})$ \\
\hline thiophenol & $1.4 \times 10^{4}$ & 70 \\
$p$-chlorothiophenol & $6.7 \times 10^{3}$ & 150 \\
$p$-thiocresol & $6.4 \times 10^{3}$ & 155 \\
\hline
\end{tabular}

Caspari and Granzow have proposed reaction (3), in which the equilibrium position had to be far to the left to account for the first-order decay kinetics ${ }^{2)}$. However, Tung and Stone ${ }^{4)}$ have raised an objection to this scheme in their recent report on the flash photolysis of aqueous solution of cysteine. They have eliminated the pseudo-first-order reaction with either $\mathrm{CySH}$ or $\mathrm{CyS}^{-}$because the decay constant was independent of cysteine concentration, and proposed the following reaction,

$$
\begin{aligned}
\mathrm{CySSCy}^{-}+\mathrm{H}_{2} \mathrm{O} & \longrightarrow \\
\mathrm{CySS} \cdot & +\mathrm{CyH}+\mathrm{OH}^{-}
\end{aligned}
$$

which involved the breaking of the $\mathrm{C}-\mathrm{S}$ bond. However, the main end product was disulfide in our product analysis by IR spectra, and benzene etc. which may be produced in the case of thiophenol according to the $\mathrm{C}-\mathrm{S}$ bond breaking could not be detected. Then, the reason for the first-order decay must be still in question.

\subsection{Assignment of the spectra}

The observed absorption bands of the disulfide anion radicals are regarded as corresponding to the transitions concerning the electrons localized on $\mathrm{S}$ atoms, for the spectra of the aliphatic disulfide anion radicals are not appreciably changed compared with those of aromatic radicals ${ }^{5)}$. However, $p$-chloro and $p$-amino substitutions for the diphenyl disulfide anion radical alter significantly the absorption maximum and the intensity of the spectrum so that the participation of $\pi$-electrons in the observed transition is sufficiently probable. More adequate assingment of the transition should be dicussed on the basis of MO calculation.

\section{References}

1) T. Takakura, M. Tagami, M. Okuyama and H. Kamada: This Journal, 24, 282 (1975).

2) G. Caspari and A. Granzow: J. Phys. Chem., 74, 836 (1970).

3) B. Dmuchovsky, F. B. Zienty and W. A. Vredenburgh: J. Org. Chem., 31, 865 (1966).

4) Tzu-Lin Tung and J.A. Stone: J. Phys. Chem., 78, 1130 (1974).

5) T. Shida: ibid., 72, 2957 (1968). 